\title{
Early Adopters of Patient-Generated Health Data Upload in an Electronic Patient Portal
}

\author{
Jessica S. Ancker ${ }^{1}$ Elizabeth Mauer ${ }^{1}$ Robin B. Kalish ${ }^{2}$ Joshua R. Vest ${ }^{3} \quad$ J. Travis Gossey ${ }^{1,4,5}$
}

${ }^{1}$ Department of Healthcare Policy and Research, Weill Cornell Medicine, New York, New York, United States

2 Department of Obstetrics and Gynecology, Weill Cornell Medicine, New York, New York, United States

${ }^{3}$ Department of Health Policy and Management, Indiana University Richard M. Fairbanks School of Health, Indianapolis, Indiana, United States

${ }^{4}$ Department of Medicine, Weill Cornell Medicine, New York, New York, United States

${ }^{5}$ Weill Cornell Physician Organization Information Services,

Weill Cornell Medicine, New York, New York, United States

Appl Clin Inform 2019;10:254-260

\section{Abstract}

Keywords

- blood glucose selfmonitoring

- self-management

- patient portals

- diabetes

- obstetricsgynecology
Address for correspondence Jessica S. Ancker, MPH, PhD, Department of Healthcare Policy and Research, Weill Cornell Medicine, 425 East 61st Street, New York, NY 10065, United States (e-mail: jsa7002@med.cornell.edu).

\section{Background and Significance}

Patient-generated health data (PGHD), that is, data recorded or generated by patients during their daily lives, could give clinicians a much more comprehensive understanding of patient health status than is available from clinical visits alone. It seems likely that a variety of types of PGHD could enrich clinical care, including exercise, activity, or diet tracking, through wearables and mobile devices ${ }^{1}$ or through self-tracked data from personal medical devices such as blood pressure and blood glucose monitors., ${ }^{2,3}$ Engaging patient in tracking and sharing PGHD could be a way to promote health knowledge, disease self-management skills, received

October 8, 2018

accepted after revision

February 17, 2019 (c) 2019 Georg Thieme Verlag KG Stuttgart · New York
DOI https://doi.org/

10.1055/s-0039-1683987. ISSN 1869-0327. 
and motivation, all of which are known to improve health outcomes. ${ }^{4-6}$

Some important barriers stand in the way of integrating PGHD into clinical practice. The use of computers, smartphones, and other health information technology remains lowest among the patients in the greatest need, including people who are elderly, less educated, or less affluent. ${ }^{7-10}$ Although some communities embrace PGHD and data tracking, other people with chronic medical conditions may be deterred from self-tracking by feelings of frustration or anxiety in response to their PGHD data, which can serve as an unwelcome reminder of their poor health. ${ }^{11}$ On the provider side, physicians who have expressed interest in potentially using PGHD (for example, in diabetes) ${ }^{12}$ still require additional support before these data are likely to be useful. Providers are likely to need practice protocols to guide clinical responses to the data, visualizations, data reduction, or decision support to help make sense of the information while preventing overload, electronic health record (EHR) integration, and integration into clinical workflow. ${ }^{3,12,13}$ Nationality studies, feasibility studies, and demonstration projects to integrate PGHD with EHRs are ongoing. . $^{4,15}$

Prior to these national studies, in 2012, our academic multispecialty practice sought to facilitate the use of PGHD by enabling an electronic data tracking tool called a flowsheet. The functionality was made available in the EHR where it could be adopted by providers as needed, but there was no specific implementation plan, encouragement, or incentivization to adopt this function. In this retrospective study, we sought to describe adoption rates and characteristics of early adopters of the PGHD functionality under these naturalistic conditions, along with preliminary data about associations with patient outcomes.

\section{Objectives}

In this retrospective study, our objectives were to describe adoption rates and characteristics of early adopters of the PGHD functionality under these naturalistic conditions and to collect preliminary data on associations with patient outcomes.

\section{Methods}

\section{Setting and Technology}

The Weill Cornell Physician Organization is the multispecialty faculty practice of Weill Cornell Medical College in New York City, representing both physician ambulatory offices and ambulatory hospital-based clinics. All physicians use the EpicCare electronic medical record and its integrated portal, which is branded as Weill Cornell Connect. Weill Cornell physicians see approximately 300,000 unique patients per year. Of the patients, $42 \%$ have electronic patient portal accounts. Providers are not salaried by Weill Cornell and participate in both public and private fee-for-service and managed-care plans. (Weill Cornell is affiliated to NewYorkPresbyterian Hospital, the largest hospital system in New
York City, but inpatient electronic systems and databases are separate from the outpatient ones and are not described in this paper.)

The flowsheet functionality was enabled in 2012 and was available to every provider. A provider selects patients for whom it appears appropriate and introduces the flowsheet to them; patients cannot initiate the use of flowsheets by themselves. After a physician enables the flowsheet for a patient, the patient can use it to upload data securely through the electronic patient portal that is accessible either through a web browser or a smartphone app. Each blood glucose value is labeled with the time, and the patient can also enter insulin dose (if any), time of the insulin administration, and free text notes. Patients may upload up to several values per day or less frequently. The physician can then view the data within the EHR in a spreadsheet format.

When this flowsheet functionality was enabled, announcements went out to all EHR users, but the tool was not attached to any specific implementation plans, research studies, or quality initiatives.

\section{Methods}

We defined flowsheet adopters as adult patients who (1) had uploaded three or more blood glucose values within any 9month period, (2) had at least a 2-year history at Weill Cornell before the first data upload, and (3) had logged into their patient portal account at least once before the first data upload. The comparison group comprised patients who had encounters with any of the physicians seen by the flowsheet adopter patients during the same time period, had any recorded diagnosis of diabetes or gestational diabetes, and had a 2-year history as well as at least one portal log-in before the date defined as the index date (see definition below). The 2-year history at Weill Cornell was chosen to ensure that there was comparable run-in data on diagnoses, clinical visits, and portal use for both flowsheet adopter and comparison patients. All variables were specified a priori, with the exception of the three-value upload threshold that was developed after inspecting the data.

The index date for the flowsheet adopter group was the date of first PGHD data upload. For the comparison group, the index date was set as the median time from between the first portal log-in and December 2016. We assessed physiological indicators (hemoglobin A1c, blood pressure, low-density lipoprotein cholesterol, and body mass index [BMI]) before and 9 months after the index date. Nine months was chosen to provide sufficient time for two to three hemoglobin A1c measurements.

The Johns Hopkins Aggregated Diagnostic Group algorithm, a case mix metric appropriate for ambulatory patients, was run on patient diagnosis codes to produce a chronic condition count. ${ }^{16}$ Patient addresses were mapped to census block to estimate patient socioeconomic status using the Centers for Disease Control's Social Vulnerability IndexSocioeconomic Status (SVI-SES) theme, a composite metric of neighborhood-level income and education levels. ${ }^{17}$ Values range from 0 to 1 , with 1 indicating the highest degree of vulnerability. 


\section{Statistical Analysis}

Categorical data were compared using chi-square tests (or Fisher's exact when cell sizes were smaller than 5). Continuous and count data were compared using independent sample $t$-tests when normally distributed or Wilcoxon's tests when skewed. Analyses were conducted using SAS v. 9.4 (SAS Institute Inc., Cary, North Carolina, United States) and R v. 3.5.0 (R Foundation, Vienna, Austria).

\section{Results}

All providers had access to the flowsheets over 4 years, including approximately 250 attending physicians in internal medicine (including endocrinologists) and approximately 40 attending physicians in obstetrics and gynecology (ob-gyn). During these 4 years, 37,578 patients had a recorded diagnosis of diabetes, 8,851 (23.5\%) of whom had portal accounts. Also, during this time, 1,395 patients had gestational diabetes, 823 (59\%) of whom had portal accounts.

Over 4 years, 16 providers chose to use the blood glucose flowsheet, and 12 of them had patients who met our inclusion criteria. Of these 12,4 were ob-gyn physicians (representing approximately $10 \%$ of the ob-gyn with access to the flowsheets), and 4 were physicians in the Department of Medicine (representing $\sim 2 \%$ of the internists with access to flowsheet). One of the internists had a specialty of endocrinology. The remaining providers who used the flowsheets were nurses in the Departments of Obstetrics and Gynecology $(n=2)$ and Medicine $(n=1)$, one individual with unknown credential in the Department of Obstetrics and Gynecology, and one individual missing both department and credential.

Fifty-three (53) patients uploaded three or more blood glucose values over any 9-month period. Of these patients, 23 were pregnant women (representing $\sim 3 \%$ of portal users with gestational diabetes) and 30 were nonpregnant adults with diabetes (representing $<1 \%$ of portal users with diabetes). A total of 201 nonpregnant comparison patients and 41 pregnant comparison patients were identified.

\section{Pregnant Patients}

The 23 pregnant patients who uploaded PGHD submitted a median of 201 (range: $23-573$ ) values to six providers in the Department of Obstetrics and Gynecology. Uploaders were similar to comparison group patients in race, ethnicity, age, and socioeconomic status assessed through both SVI-SES and insurance type (-Table 1). However, uploaders had more clinical encounters and portal log-ins before initial data upload, somewhat earlier establishment of patient portal accounts, and worse baseline blood pressure.

For pregnant patients, average BMI dropped significantly more in the 9 months after the index date (i.e., post-partum) among uploaders than nonuploaders. However, average blood pressure increased more among uploaders than nonuploaders.

\section{Chronic Disease Patients}

A total of 30 nonpregnant patients with diabetes diagnoses uploaded a median of $139.5(15-1,253)$ values to 10 provi- ders in the departments of Internal Medicine, Endocrinology, Cardiology, and Nutrition.

Among these chronic disease patients, uploaders were more likely than comparison group patients to be AsianAmerican and were younger, but the groups did not have other significant demographic differences including in socioeconomic status ( - Table 1 ). Uploaders had more clinical visits and portal log-ins before initial data upload.

During the 9-month period after the first glucose value upload, uploaders had significantly larger reductions in hemoglobin A1C and BMI than did nonuploaders (-Table 1).

Uploaders also experienced large variations in hemoglobin A1c level after the index date ( - Fig. 1). Uploaders appeared to fall in two subsets (-Fig. 2). One subset had low, wellcontrolled hemoglobin A1c values both before and during PGHD upload. A second subset began upload at a time when their hemoglobin A1c was elevated and typically experienced a hemoglobin A1c decrease followed by a plateau.

\section{Discussion}

Self-tracked or PGHD appears to offer the possibility of improved clinical monitoring for patients between clinical visits. Many patients who track data would like their physicians to review it to identify health problems or reassure them that all is well. Nevertheless, in an academic multispecialty practice with high rates of patient portal use, adoption of a PGHD data upload function has been slow. Over 4 years, 16 providers tried it, and 53 established patients uploaded three or more values.

The most marked difference between uploaders and comparison patients was that uploaders had more visits and portal log-ins. However, uploaders did not have more chronic conditions than nonuploaders and did not have markedly worse baseline physiological indicators (with the exception of higher systolic blood pressure among the pregnant subgroup). Overall, this suggests that uploaders were not substantively sicker than nonuploaders but rather more engaged in their health care, motivated to change behavior, or loyal to the medical center. They may also have been more facile with information technology, a point supported by the fact that uploaders among the chronic disease patients were younger than nonuploaders. Patient portal use has been shown to be associated with socioeconomic status, with more affluent patients having higher average health literacy and better access to computers, broadband internet, and smartphones. ${ }^{8,18-21}$ However, in our study, we included only patients with portal accounts and a history of using them. Among these patients, there were no socioeconomic differences between uploaders and nonuploaders, as indicated by insurance type or a composite measure of U.S. Census tract-level income and education.

Among the chronic disease patients, PGHD uploading was associated with marked variation in hemoglobin A1c values (-Fig. 1) and significant reductions in hemoglobin A1c and BMI over a 9-month period ( - Table 1 ). It appears that uploading was associated with a period of medical transition or change. However, given the baseline differences between the two groups, it would be difficult to conclude that the uploading behavior caused the reduction. 
Table 1 Characteristics of patient-generated health data uploaders and comparison with patients who did not upload

\begin{tabular}{|c|c|c|c|c|c|c|c|c|c|c|c|}
\hline & & \multicolumn{5}{|c|}{ Chronic disease patients } & \multicolumn{5}{|c|}{ Pregnant women } \\
\hline & & \multicolumn{2}{|c|}{$\begin{array}{l}\text { Uploaders } \\
(n=30)\end{array}$} & \multicolumn{2}{|c|}{$\begin{array}{l}\text { Comparison } \\
\text { patients } \\
(n=201)\end{array}$} & \multirow{2}{*}{$\begin{array}{l}p \text {-Value } \\
0.05\end{array}$} & \multicolumn{2}{|c|}{$\begin{array}{l}\text { Uploaders } \\
(n=23)\end{array}$} & \multicolumn{2}{|c|}{$\begin{array}{l}\text { Comparison } \\
\text { patients } \\
(n=41)\end{array}$} & \multirow{2}{*}{$\begin{array}{l}p \text {-Value } \\
0.94\end{array}$} \\
\hline \multirow[t]{4}{*}{ Race } & White & 14 & $46.7 \%$ & 119 & $59.2 \%$ & & 14 & $60.9 \%$ & 24 & $58.5 \%$ & \\
\hline & Black & 1 & $3.3 \%$ & 8 & $4 \%$ & & 0 & $0 \%$ & 0 & $0 \%$ & \\
\hline & Asian & 7 & $23.3 \%$ & 24 & $11.9 \%$ & & 6 & $26.1 \%$ & 10 & $24.4 \%$ & \\
\hline & All other & 8 & $26.7 \%$ & 50 & $24.9 \%$ & & 3 & $13 \%$ & 7 & $17.1 \%$ & \\
\hline \multirow[t]{3}{*}{ Ethnicity } & Hispanic & 4 & $13.3 \%$ & 16 & $8 \%$ & 0.28 & 2 & $8.7 \%$ & 4 & $9.8 \%$ & $>0.99$ \\
\hline & Not Hispanic & 24 & $80 \%$ & 146 & $72.6 \%$ & & 20 & $87 \%$ & 34 & $82.9 \%$ & \\
\hline & All other & 2 & $6.7 \%$ & 39 & $19.4 \%$ & & 1 & $4.3 \%$ & 3 & $7.3 \%$ & \\
\hline Age & & \multicolumn{2}{|c|}{$39.2(14.4)$} & \multicolumn{2}{|c|}{$47(15.2)$} & 0.004 & \multicolumn{2}{|c|}{$31.1(4.5)$} & \multicolumn{2}{|c|}{$29.9(4.5)$} & 0.37 \\
\hline \multirow[t]{4}{*}{ Payer } & Commercial & 26 & $86.7 \%$ & 136 & $67.7 \%$ & 0.48 & 20 & $87 \%$ & 36 & $87.8 \%$ & 0.79 \\
\hline & Medicaid & 0 & $0 \%$ & 3 & $1.5 \%$ & & 0 & $0 \%$ & 1 & $2.4 \%$ & \\
\hline & Unknown & 1 & $3.3 \%$ & 16 & $8 \%$ & & 3 & $13 \%$ & 3 & $7.3 \%$ & \\
\hline & Medicare & 3 & $10 \%$ & 45 & $22.4 \%$ & & 0 & $0 \%$ & 0 & $0 \%$ & \\
\hline \multicolumn{2}{|c|}{ Chronic condition count } & \multicolumn{2}{|c|}{$5.9(4.7)$} & \multicolumn{2}{|c|}{$6(4.5)$} & 0.92 & \multicolumn{2}{|c|}{$2.4(1.9)$} & \multicolumn{2}{|c|}{$1.1(0.9)$} & 0.10 \\
\hline SVI-SES & & \multicolumn{2}{|c|}{$0.27(0.30)$} & \multicolumn{2}{|c|}{$0.24(0.26)$} & 0.63 & \multicolumn{2}{|c|}{$3(2.6)$} & \multicolumn{2}{|c|}{$1.9(2.2)$} & 0.62 \\
\hline \multicolumn{2}{|c|}{$\begin{array}{l}\text { Encounters year before } \\
\text { index }\end{array}$} & \multicolumn{2}{|c|}{$10.2(4.6)$} & \multicolumn{2}{|c|}{$6.5(5.3)$} & $<0.001$ & \multicolumn{2}{|c|}{$14(9.1)$} & 8.1 & & 0.003 \\
\hline $\begin{array}{l}\text { Portal log } \\
\text { index }\end{array}$ & ear before & 65. & 47.6) & 23.5 & 8.1) & $<0.001$ & 65.4 & 44.7) & 26.2 & 34.3) & $<0.001$ \\
\hline Year of fir & ncounter & 2,00 & (3.3) & 2,008 & 3.1) & 0.18 & 2,00 & (3) & 2,01 & (2.7) & 0.08 \\
\hline $\begin{array}{l}\text { Year estat } \\
\text { account }\end{array}$ & ed portal & 2,0 & (1.6) & 2,012 & 1.9) & 0.10 & 2,01 & (1.9) & 2,01 & (1.6) & 0.004 \\
\hline Baseline $\mathrm{h}$ & globin A1c (\%) & 7.8 & & $6.8(1$ & & 0.17 & 5.5 & & 5.7 & & 0.54 \\
\hline $\begin{array}{l}\text { Baseline s } \\
\text { pressure }\end{array}$ & lic blood & 117 & (13) & 118.5 & 16) & 0.64 & 109 & & 104. & (6.6) & 0.04 \\
\hline $\begin{array}{l}\text { Baseline } \\
\text { pressure }\end{array}$ & lic blood & 72.3 & 10.5) & 71.8 & & 0.82 & 66.7 & 6.8) & 65( & & 0.33 \\
\hline Baseline L & & 118 & $(38.1)$ & 101.1 & 33.2) & 0.06 & 102. & $(36.3)$ & 102 & 7.3) & 0.95 \\
\hline Baseline b & mass index & 29.2 & $6.8)$ & 28.2 & .2) & 0.46 & 27.2 & 5.6) & 24.7 & 3.8) & 0.06 \\
\hline $\begin{array}{l}\text { Change in } \\
\text { A1c (\%) }\end{array}$ & noglobin & $-0 . \varepsilon$ & 1.9) & $0.1(C$ & & 0.02 & $N A^{a}$ & & $N A^{a}$ & & - \\
\hline $\begin{array}{l}\text { Change in } \\
\text { pressure }\end{array}$ & tolic blood & 5.5 & 6.7) & $1.6(1$ & & 0.46 & 7.3 & & 0.4 & & 0.04 \\
\hline $\begin{array}{l}\text { Change in } \\
\text { pressure }\end{array}$ & tolic blood & 3.8 & & $0.9(\mathrm{c}$ & & 0.38 & 5.8 & $0.1)$ & -2.6 & 7.4) & 0.02 \\
\hline Change in & & 2.1 & 3.5) & $0.1(2$ & $.9)$ & 0.28 & $N A^{a}$ & & $N A^{a}$ & & - \\
\hline Change in & dy mass index & -1. & 2.2) & $0.2(1$ & & 0.01 & -3.1 & $3.2)$ & 0.8 & & 0.003 \\
\hline
\end{tabular}

Abbreviations: LDL, low-density lipoprotein; NA, not available; SVI-SES, Centers for Disease Control's Social Vulnerability Index-Socioeconomic Status. ${ }^{a}$ As these indicators are not typically monitored multiple times during pregnancy, fewer than 10 pregnant patients had both baseline and follow-up values for $L D L$ and hemoglobin A1c.

Note: $p$-values of 0.05 or lower are shown in boldface.

Among pregnant patients, BMI is expected to rise during pregnancy, but we found that after birth, BMI dropped more among uploaders than nonuploaders. By contrast, blood pressure increased more among uploaders than comparison patients. This could be an artifact of the fact that PGHD patients were slightly sicker (more than two comorbidities compared with approximately $1 ; p=0.1$ ) or that some patients with gestational diabetes may have had comorbid preeclampsia. Hemoglobin A1c is not typically monitored during pregnancy, and therefore there were little data on this 


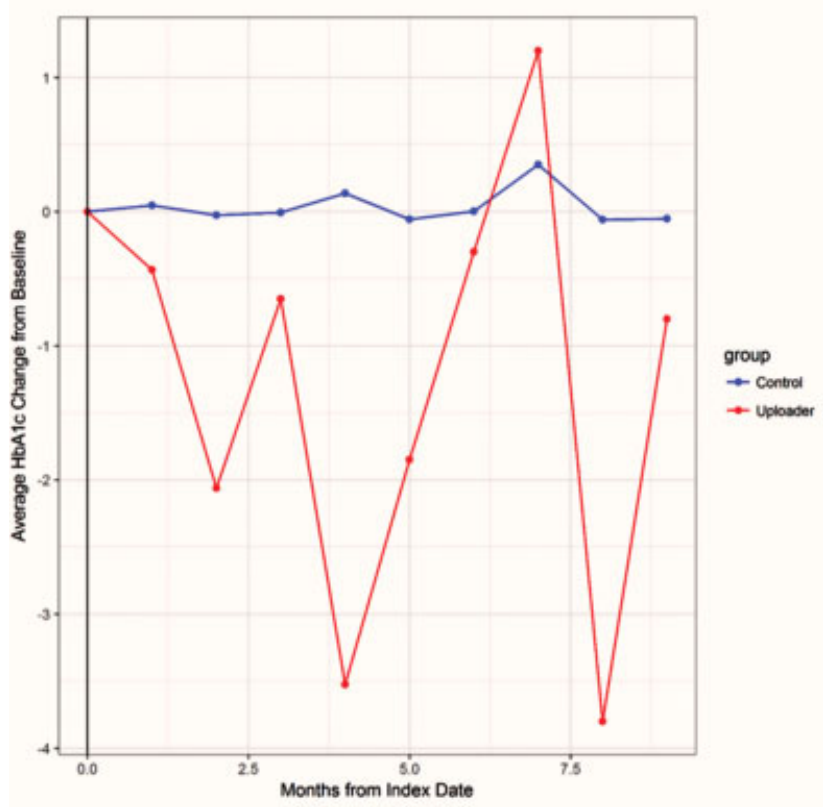

Fig. 1 Average change in hemoglobin A1c values among chronic disease patients who did and did not upload glucose data. Chronic disease patients who uploaded patient-generated health data had more variable hemoglobin A1c values over 9 months than comparison patients. The index date in the graph is the date of the first upload for uploaders and the median time since initial portal log-in for comparison patients.

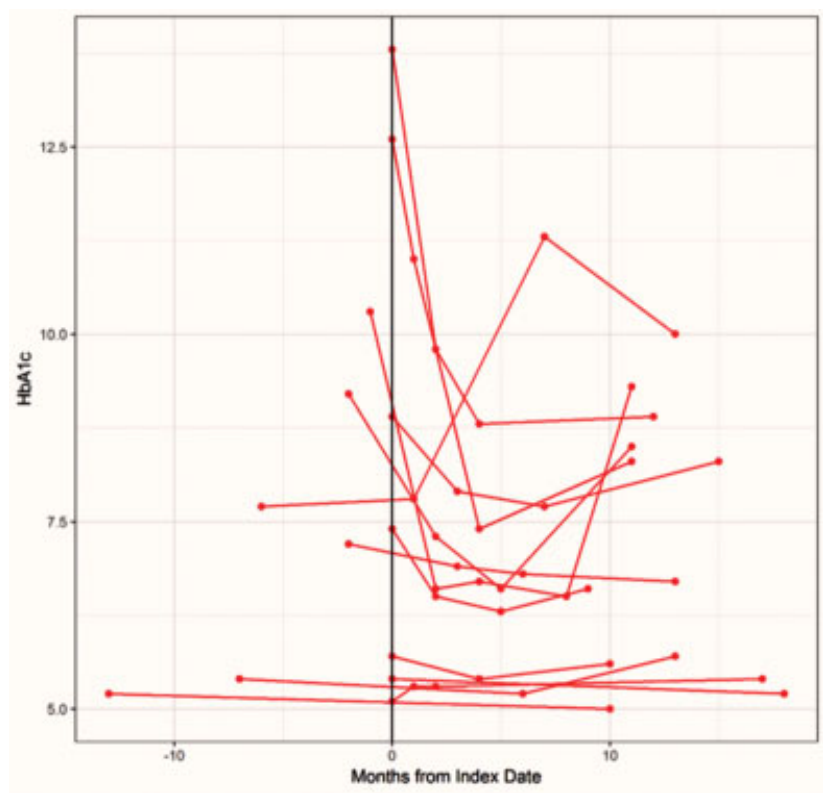

Fig. 2 Hemoglobin A1c (HA1c) values among chronic disease patients who uploaded patient-generated health data. Among chronic disease patients, uploaders were clustered into two groups. The first (bottom) had well-controlled HA1c values at the time of the first upload (index date) and maintained low values throughout. The second began uploading at a time of HA1c elevation and, with one exception, saw reductions after beginning to upload.

end point. Again, the baseline differences between the two groups, especially in terms of patient portal use, mean that the observed effects were associated with the flowsheet but may not have been caused by it.
The low adoption rate for PGHD upload is probably attributable to several factors. The first factor is barriers to provider uptake. It is widely accepted that integration into the EHR is a prerequisite for the use of novel tools, and, in other situations, lack of integration is cited as a barrier to adoption. ${ }^{3,22}$ However, even though this PGHD upload tool was integrated into the EHR, providers would still have had to select patients for whom it would be appropriate, determine if the patients are interested and train them to use it, and then would presumably feel responsible for checking uploaded values regularly to provide feedback. In our center, rollout of the new function was announced across the institution but was not accompanied by a financial or other incentive program that would have covered the time costs of online monitoring or by an implementation plan with clinical champions to promote use. In this naturalistic situation, providers who adopted the new function were those who were already intensively monitoring patients. This included physicians and nurses in the Department of Obstetrics and Gynecology, which has protocols for routine screening for gestational diabetes and preeclampsia at 24 to 28 weeks of gestation. For women with high-risk pregnancies, physicians promoted daily self-monitoring even before the electronic PGHD tool was enabled. Patient-recorded values are typically checked regularly by a physician or a nurse, and patients are encouraged to call when values are concerning. By contrast, there is no standardized approach to PGHD data upload for patients with chronic diabetes; therefore, uptake and workflow depend upon provider preference. In this group, a small number of providers used the flowsheets for patients at a time of hemoglobin A1c elevation, which may have been a time when patients were motivated to attempt lifestyle changes or when providers were making and monitoring changes in medical management. Others have noted that even providers who recognize the value of PGHD still express the need for support such as practice protocols. ${ }^{3}$

A second explanation for low adoption rates is that PGHD upload is likely to require active and continued buy-in from patients. Our inclusion criteria specified that patients must already have a patient portal account. However, it is also likely that PGHD would be adopted only by patients who were willing to measure and input glucose values regularly and to check provider feedback through portal messaging. ${ }^{23}$ Self-monitoring of blood glucose can be challenging both physically and emotionally. In some cases, patients may find self-monitoring helpful in understanding the impact of their lifestyle and increasing their sense of control. However, in others, it may lead to feelings of burden, frustration, anxiety, or self-blame, especially when values are not "good" or when trends do not clearly link to lifestyle changes. ${ }^{11,24}$ This is likely to reduce the numbers of patients willing to commit to routine PGHD upload. It may also lead to abandonment after trying the tool. In many domains, people abandon personal tracking tools after they have satisfied their curiosity, when their health status or life circumstances change, or when tracking starts to become burdensome. ${ }^{25,26}$ In other clinical domains, sustained engagement with self-monitoring has also been linked to strong relationships with the health care 
provider, supportive environments, and regular feedback. ${ }^{23}$ In our study, another probable barrier was the need for manual data input. Although, in theory, patients could upload glucose values directly from monitors through Apple Healthkit, the data did not allow us to determine how many patients took advantage of this potential convenience, and it is not clear how easy it was for them to do this. In the future, widespread integration of PGHD into clinical care will likely depend upon (1) targeted implementation strategies including measures to ensure that physicians are fully incentivized to collect and review remotely collected PGHD data rather than prioritizing face-to-face visits, (2) modifications that make technologies easier for patients to use, such as by eliminating manual data input, (3) disease- or populationspecific decision support that uses the PGHD, ${ }^{14}$ and (4) a stronger evidence base demonstrating ways in which the time and energy needed to generate, review, and provide feedback on PGHD measurably improve health, satisfaction, or other important outcomes. This will be important to ensure that patients are helped to track information of personal value to them rather than data to be used to meet quality measurement or value-based purchasing initiatives.

\section{Limitations}

The study is limited by its small sample size and unmeasured confounders such as health care engagement, literacy, patient activation, and motivation, which could account for differences between uploaders and nonuploaders. The sample size meant that it was not feasible to apply causal inference methods such as propensity score matching. Because of these problems, we also avoided conducting regression analysis. This makes it impossible to rule out the possibility that the confounders (engagement with health care and patient age) could be responsible for the observed improvement in patient outcomes. The heterogeneity of the settings and of the patients and clinicians in this particular dataset also makes it challenging to determine whether this technology might be appropriate for certain subsets. Targeted studies with selected patient populations would be needed to develop estimates of efficacy of this technology; the current analysis of this natural experiment is useful only for showing its limited effectiveness in this specific setting. Also, this short-term analysis does not provide an evidence on long-term effects or sustainability.

\section{Conclusion}

Despite the potential for PGHD to help medical monitoring of patients between visits, adoption of a PGHD function within an academic multispecialty practice was slow. The function appeared to be adopted by providers who sought to monitor patients in a period of medical instability or transition, such as, women during high-risk pregnancy and chronic disease patients after an elevated hemoglobin A1c measurement. Patients who uploaded PGHD had more visits and portal uploads than patients who did not, but they did not appear markedly sicker than nonuploaders, suggesting that they may have been more engaged with their health care or motivated. Chronic disease patients who uploaded PGHD data experienced improvements in their hemoglobin A1c and BMI values. However, the improvement may have been because of motivation or increased engagement, meaning that PGHD upload may be a support tool for patients who are already motivated rather than one that directly improves outcomes.

\section{Clinical Relevance Statement}

Self-tracked or PGHD appears to offer the possibility of improved clinical monitoring for patients between clinical visits and may be associated with improved outcomes. Nevertheless, physicians may be slow to embrace PGHD data upload to the EHR unless the process is streamlined, incentivized or reimbursed, or promoted through targeted implementation efforts.

\section{Multiple Choice Questions}

1. Diabetes patients who uploaded PGHD to a patient portal showed improvement in their hemoglobin A1c values. Nevertheless, the effect might be because of the following:

a. Higher socioeconomic status among these patients.

b. Fewer clinical visits among these patients.

c. Higher health care utilization among these patients.

d. Improved medication safety among the patient.

Correct Answer: The correct answer is option c, higher health care utilization among these patients. Although the patients did have improved hemoglobin A1c, they also were more frequent users of the portal and more frequent users of the health care system than nonuploaders. This could be the explanation for their improved outcomes. By contrast, their neighborhood-level socioeconomic status was tested and did not show any significant difference; therefore, this is unlikely to be the explanation for the findings.

2. Patient-generated health data (PGHD) is a term that encompasses which of the following types of information: a. Patients answering questions about the quality of their health care.

b. Patients tracking health indicators of interest to themselves.

c. Patients describing their own health status.

d. Patient satisfaction.

Correct Answer: The best answer is option $b$, patients tracking health indicators of interest to themselves. The other options (a, c, and d) are instead considered patientreported outcomes.

Protection of Human and Animal Subjects

This study was approved by the Weill Cornell Institutional Review Board with a waiver of consent for use of deidentified data.

Funding

This project was funded by the Agency for Healthcare Research and Quality (K01 HS021531; PI: Ancker). 
Conflict of Interest

None declared.

\section{References}

1 Say P, Stein DM, Ancker JS, Hsieh CK, Pollak JP, Estrin D. Smartphone data in rheumatoid arthritis - what do rheumatologists want? AMIA Annu Symp Proc 2015;2015:1130-1139

2 American Association of Diabetes Educators. The American Association of Diabetes Educators position statement: self-monitoring of blood glucose using glucose meters in the management of type 2 diabetes. 2010 copyright, Chicago, IL: American Association of Diabetes Educators. Available at: https://www.diabeteseducator. org/docs/default-source/practice/practice-documents/practicepapers/smbg-using-glucose-meters-in-management-of-t2d.pdf? sfvrsn $=0$. Accessed March 18, 2019.

3 Cohen DJ, Keller SR, Hayes GR, Dorr DA, Ash JS, Sittig DF. Integrating patient-generated health data into clinical care settings or clinical decision-making: lessons learned from Project HealthDesign. JMIR Human Factors 2016;3(02):e26

4 Greenhalgh T. Patient and public involvement in chronic illness: beyond the expert patient. BMJ 2009;338(7695):b49

5 Hibbard JH, Greene J. What the evidence shows about patient activation: better health outcomes and care experiences; fewer data on costs. Health Aff (Millwood) 2013;32(02):207-214

6 Hibbard JH, Mahoney ER, Stock R, Tusler M. Do increases in patient activation result in improved self-management behaviors? Health Serv Res 2007;42(04):1443-1463

7 Fox S, Duggan M. Pew Research Center: Internet \& Technology. Mobile Health 2012. 2013. Available at: http://www.pewinternet. org/2012/11/08/mobile-health-2012/. Accessed March 13, 2019

8 Ancker JS, Barrón Y, Rockoff ML, et al. Use of an electronic patient portal among disadvantaged populations. J Gen Intern Med 2011; 26(10):1117-1123

9 Roblin DW, Houston TK II, Allison JJ, Joski PJ, Becker ER. Disparities in use of a personal health record in a managed care organization. J Am Med Inform Assoc 2009;16(05):683-689

10 Veinot TC, Mitchell H, Ancker JS. Good intentions are not enough: how informatics interventions can worsen inequality. J Am Med Inform Assoc 2018;25(08):1080-1088

11 Ancker JS, Witteman HO, Hafeez B, Provencher T, Van de Graaf M, Wei E. "You get reminded you're a sick person": personal data tracking and patients with multiple chronic conditions. J Med Internet Res 2015;17(08):e202

12 Nundy S, Lu C-YE, Hogan P, Mishra A, Peek ME. Using patientgenerated health data from mobile technologies for diabetes selfmanagement support: provider perspectives from an academic medical center. J Diabetes Sci Technol 2014;8(01):74-82

13 West P, Kleek MV, Giordano R, Weal MJ, Shadbolt N. Common Barriers to the Use of Patient-Generated Data across Clinical
Settings. Proceedings of the 2018 CHI Conference on Human Factors in Computing Systems, Montreal, Quebec, Canada, 2018

$14 \mathrm{Lv}$ N, Xiao L, Simmons ML, Rosas LG, Chan A, Entwistle M. Personalized hypertension management using patient-generated health data integrated with electronic health records (EMPOWER-H): six-month pre-post study. J Med Internet Res 2017;19(09):e311

15 Office of the National Coordinator for Health Information Technology. A practical approach to the use of patient-generated health data (PGHD). Available at: https://www.healthit.gov/ sites/default/files/onc_pghd_pilot_demonstrations_infographic. pdf. Accessed March 13, 2019

16 Weiner JP, Starfield BH, Steinwachs DM, Mumford LM. Development and application of a population-oriented measure of ambulatory care case-mix. Med Care 1991;29(05):452-472

17 Flanagan BE, Gregory EW, Hallisey EJ, Heitgerd JL, Lewis B. A social vulnerability index for disaster management. J Homel Secur Emerg Manag 2011;8(Suppl 1):1-24

18 Sarkar U, Karter AJ, Liu JY, et al. The literacy divide: health literacy and the use of an internet-based patient portal in an integrated health system-results from the diabetes study of northern California (DISTANCE). J Health Commun 2010;15 (Suppl 2):183-196

19 Sarkar U, Karter AJ, Liu JY, et al. Social disparities in internet patient portal use in diabetes: evidence that the digital divide extends beyond access. J Am Med Inform Assoc 2011;18(03): 318-321

20 Rainie L. Digital Divides 2016. Washington, DC: Pew Research Center; 2016

21 Zickuhr K. Who's Not Online and Why. Washington, DC: Pew Research Center; 2013

22 Prey JE, Polubriaginof F, Grossman LV, et al. Engaging hospital patients in the medication reconciliation process using tablet computers. J Am Med Inform Assoc 2018;25(11):1460-1469

23 Reading M, Baik D, Beauchemin M, Hickey KT, Merrill JA. factors influencing sustained engagement with ECG self-monitoring: perspectives from patients and health care providers. Appl Clin Inform 2018;9(04):772-781

24 Peel E, Parry O, Douglas M, Lawton J. Blood glucose self-monitoring in non-insulin-treated type 2 diabetes: a qualitative study of patients' perspectives. Br J Gen Pract 2004;54(500):183-188

25 Epstein DA, Caraway M, Johnston C, Ping A, Fogarty J, Munson SA. Beyond abandonment to next steps: understanding and designing for life after personal informatics tool use. Proc SIGCHI Conf Hum Factor Comput Syst 2016;2016:1109-1113

26 Clawson J, Pater JA, Miller AD, Mynatt E, Mamykina L No longer wearing: investigating the abandonment of personal health-tracking technologies on craigslist. Paper presented at the UbiComp '15 Proceedings of the 2015 ACM International Joint Conference on Pervasive and Ubiquitous Computing, September 7-11, 2015, Osaka, Japan 